Bundesgesundheitsbl 2013 · 56:1597-1599

DOI 10.1007/s00103-013-1861-8

Online publiziert: 24. November 2013

(c) Springer-Verlag Berlin Heidelberg 2013
H. Tönnies · L. Schaade

Robert Koch-Institut, Berlin

\section{Sexuelle und reproduktive Gesundheit}

\section{Medizinisch-naturwissenschaftliche Aspekte}

Liebe Leserinnen und Leser,

nach dem Erscheinen des ersten Bandes „Sexuelle und reproduktive Gesundheit" im Frühjahr dieses Jahres, der sich vor allem den diesbezüglichen sozialwissenschaftlichen Aspekten widmete, halten Sie hier nun den zweiten Band zum Thema in den Händen, der gezielt auf die medizinisch-naturwissenschaftlichen Aspekte der sexuellen und reproduktiven Gesundheit eingeht.

Die medizinisch-naturwissenschaftlichen Aspekte der sexuellen und reproduktiven Gesundheit sind vielfältig, was sich auch in den verschiedenen Themenbereichen, die in diesem Schwerpunktheft behandelt werden, widerspiegelt. $\mathrm{Zu}$ nennen sind hier die sexuell übertragbaren Infektionen (STI), HIV/Aids und Fragestellungen zur Fertilität, Infertilität und Pränatal- und Präimplantationsdiagnostik.

Unser heutiges Wissen zur sexuellen und reproduktiven Gesundheit basiert sowohl auf sozialwissenschaftlichen als auch auf medizinisch-naturwissenschaftlichen Erkenntnissen. Sexuelle und reproduktive Gesundheit sind ein integraler Bestandteil von Lebensqualität. Es ist daher allgemeiner Konsens, dass ein direkter Zugang aller zu umfassenden Informationen und Angeboten im Bereich der sexuellen und reproduktiven $\mathrm{Ge}$ sundheit unerlässlich ist. Nur dieser gewährleistet eine positive und respektvolle Einstellung gegenüber sexueller und reproduktiver Gesundheit.
Die ersten beiden Beiträge dieser Ausgabe beschäftigen sich vor allem mit der Situation von Männern, die Sex mit Männern haben (MSM). Es ist gut belegt, dass persistierende STI das Pro-Kontakt-Risiko einer HIV-Infektion erhöhen. Die aktuellen epidemiologischen Zahlen zeigen, dass die Prävention von STI und HIV ein zentrales Thema der sexuellen Gesundheit darstellt. O. Hamouda beschreibt in seinem Beitrag die epidemiologischen Entwicklungen bei ausgewählten übertragbaren Infektionen wie Syphilis und Gonorrhö in Verbindung mit dem Risiko einer HIV-Infektion. Er macht deutlich, dass es dringend verstärkter Anstrengungen durch Kombination der zur Verfügung stehenden Möglichkeiten bedarf, um die Zahl der Neuinfektionen mit HIV einzudämmen und zeitgleich STI zu verhindern.

A.J. Schmidt und U. Marcus weisen in ihrem Artikel ebenfalls auf bestehende Defizite sowohl in der Zahl der Angebote als auch in der inhaltlichen Angebotsbreite zur STI-Diagnostik für Männer mit gleichgeschlechtlichen Sexualkontakten vor allem in deutschsprachigen Städten hin. Sie belegen ihre Schlussfolgerung anhand von aktuellen Daten, die im Rahmen des Europäischen MSM-InternetSurveys (EMIS) in 38 Ländern ermittelt wurden. Die Autoren weisen darauf hin, dass die Unterdiagnose rektaler STI bei MSM im deutschsprachigen Raum direkte Auswirkungen auf die individuelle sexuelle Gesundheit, HIV-Prävention und nicht zuletzt Vergleichbarkeit euro- päischer Surveillance-Daten hat. Sie sprechen sich für niedrigschwellige und den sexuellen Praktiken von MSM angepasste Untersuchungsangebote aus, um die sexuelle Gesundheit von MSM nachhaltig zu verbessern.

Sexuell übertragene Krankheiten (STD) und Infektionen stellen neben einer Vielfalt von anderen medizinischen Aspekten eine bekannte Ursache für die männliche und weibliche Infertilität dar. E. Nieschlag fasst den aktuellen medizinischen Kenntnisstand zur Infertilität bei Männern zusammen. Trotz aller pathophysiologischen und methodischen Fortschritte in der Andrologie und der Tatsache, dass bei der Hälfte der Paare mit unerfülltem Kinderwunsch der Mann Störungen der Fortpflanzungsfähigkeit aufweist, wird aus Sicht des Autors noch heute die angewandte Diagnostik und zielgerichtete Behandlung des fertilitätsgestörten Mannes vernachlässigt. Auch wenn für etwa ein Drittel der männlichen Fertilitätsstörungen keine rationalen Behandlungsmöglichkeiten existieren, helfen häufig zur Erfüllung des Kinderwunsches Techniken der assistierten Reproduktion (z. B. IVF, ICSI; s. auch Beitrag von H. Kentenich, C. Sibold, A. TandlerSchneider). Präventive Maßnahmen wie die frühzeitige Behandlung eines Hodenhochstands im Säuglingsalter oder Änderungen des Lebensstils (z. B. Einstellung des Rauchens) können Infertilität verhindern. Der Autor schließt mit der Feststellung, dass Fertilitätsprobleme aufseiten des Mannes häufig auch durch die 
Optimierung der weiblichen reproduktiven Funktion kompensiert werden können. Hier schließen sich die beiden weiteren Artikel nahtlos an. Der Aufsatz von T. Strowitzki fokussiert auf die Ursachen der weiblichen Infertilität und diesbezügliche neueste Therapieoptionen. Die Ursachen für die Infertilität bei Frauen sind ebenso vielfältig wie beim männlichen Geschlecht und umfassen neben Infektionen, Umweltfaktoren und dem Lebensstil endokrinologische, metabolische und anatomisch-organische Veränderungen/Störungen. Aktuell zielt die Behandlung weiblicher Infertilität statt auf Maßnahmen der assistierten Reproduktion wieder vermehrt auf die Wiederherstellung der eigenen Fertilität durch die gezielte Diagnostik und Therapie z. B. metabolischer Erkrankungen (vor allem Schilddrüsen- und Zuckerstoffwechsel) oder mittels Fertilitätschirurgie.

Von der Seite der natürlichen Fertilität des Paares und den epidemiologischen Aspekten der Subfertilität nähert sich C. Gnoth dem Thema der reproduktiven Gesundheit. Er stellt die anhand einer aktuellen Literaturrecherche gewonnenen Daten zur natürlichen Fertilität und zur Epidemiologie der Subfertilität dar und verdeutlicht auf ihrer Basis auch die Bedeutung der neuen, sog. perikonzeptionellen Medizin, die mit Blick auf die reproduktive Gesundheit eines Paares (Erhalt der Fertilität, Prävention und Management von Schwangerschaftsrisiken) verschiedene medizinische Fachdisziplinen zusammenführt. Er stellt die „Fertility awareness“ mittels Gewahrwerdung, Wahrnehmung und Beobachtung der Fertilität durch das Paar selbst zur Optimierung der Konzeptionsaussichten bei Kinderwunsch als eine wichtige Methode vor.

Dass der interdisziplinäre Austausch der ärztlichen Fachdisziplinen Humangenetik, Gynäkologie und Reproduktionsmedizin im Bereich der reproduktiven Gesundheit eine zentrale Rolle spielt, zeigen auch die folgenden Artikel zu genetischen Aspekten der Fertilitätsstörungen (P. Wieacker), zur In-vitro-Fertilisation (H. Kentenich, C. Sibold, A. Tandler-Schneider), zur Pränatal- (M. Stumm, M. Entezami) und Präimplantationsdiagnostik (M. Montag, B. Toth, T. Strowitz- ki). Vor dem Hintergrund genetischer Ursachen referiert P. Wieacker den aktuellen Wissensstand zu den Störungen der Fertilität bei Frauen und Männern. Chromosomenaberrationen (Aneuploidien, strukturelle Aberrationen) können ebenso Ursache für Fertilitätsstörungen sein wie monogene Defekte. Störungen der Fertilität können durch Störungen der Gonadendifferenzierung, gonadale Funktionsstörungen, Beeinträchtigung der Keimzellproduktion oder Anomalien der Genitalwege bedingt sein. Der Beitrag gibt einen umfassenden Überblick über die genetischen Aspekte der Fertilitätsstörungen. Anhand der dargestellten genetischen Ursachen für Fertilitätsstörungen wird deutlich, wie komplex die vielfältigen Mechanismen im Reproduktionsgefüge des Menschen ineinandergreifen.

Als ein Ausweg aus ungewollter Kinderlosigkeit können für einen Teil der Betroffenen die reproduktionsmedizinischen Verfahren der In-vitro-Fertilisation und der intrazytoplasmatischen Spermieninjektion - beides Verfahren der extrakorporalen Befruchtung - hilfreich sein. A. Kentenich, C. Sibold und A. Tandler-Schneider stellen den aktuellen medizinisch-wissenschaftlichen Stand dieser - für die Betroffenen teils recht kostspieligen - Sterilitätstherapie zur Erfüllung eines Kinderwunsches ausführlich in ihrem Artikel dar. Grundsätzlich nimmt der Beratungsaspekt in der Kinderwunschbehandlung eine hervorgehobene Rolle ein. Die Autoren stellen die Erfolgsaussichten und Probleme (z. B. ungewollte Mehrlingsschwangerschaften) der Verfahren dar und benennen neben der wissenschaftlichen Evidenz ebenso medizinische, psychologische und ethische Aspekte, basierend auf den aktuellen rechtlichen Möglichkeiten in Deutschland.

Die Pränataldiagnostik mit ihren bildgebenden, nichtinvasiven Verfahren (Ultraschalluntersuchung) als auch die invasive genetische Pränataldiagnostik sind Inhalt des folgenden Artikels von M. Stumm und M. Entezami. Die Entwicklungen in der medizinischen Forschung und Technik der letzten Jahre haben die Pränataldiagnostik wie das gesamte Gebiet der molekularen Medizin nachdrücklich verändert. Die Bedeutung der ultraschallbasierten Screeninguntersuchungen sowie der detaillierten Fehlbildungsdiagnostik werden in dem Artikel ebenso beleuchtet wie die in den letzten Jahren rückläufige Inanspruchnahme invasiver pränataler Untersuchungen als „Standard“. Auch werden neueste Entwicklungen wie die erst seit Kurzem anwendbaren nichtinvasiven pränatalen Testmethoden mit ihren möglichen Vorund Nachteilen dargestellt. Die Vielfalt der verschiedenen Untersuchungsoptionen macht einmal mehr deutlich, welch hohen Stellenwert eine adäquate genetische Beratung vor Inanspruchnahme einer solchen Untersuchung hat.

M. Montag, B. Toth und T. Strowitzki stellen die aktuellen Möglichkeiten der Präimplantationsdiagnostik (PID) vor. Schon seit einiger Zeit in Deutschland etabliert, ermöglicht die PID im Rahmen der Polkörperdiagnostik Untersuchungen auf maternal vererbte numerische und strukturelle Chromosomenveränderungen sowie die gezielte Analyse auf bekannte monogene Eigenschaften. Die Autoren beleuchten ebenso die aktuellen Voraussetzungen und Entwicklungen zur Throphektodermbiopsie und genetischen Diagnostik unter Berücksichtigung der Änderung des Embryonenschutzgesetzes in Deutschland. Während die Umsetzung der PID in Deutschland noch in der Etablierungsphase ist, wird sie weltweit seit nunmehr fast 2 Jahrzehnten praktiziert. Die daraus resultierenden Erfahrungen sind Bestandteil des vorliegenden Beitrages.

K. Vetter und M. Gockenjan widmen sich ausführlich dem Thema der Schwangerenvorsorge in Deutschland als flächendeckendes, standardisiertes Programm zur optimierten Betreuung der Schwangeren. Auch hier spielen die Information, Aufklärung und Beratung der Schwangeren eine wesentliche Rolle. Prinzip der Schwangerenvorsorge ist, wie eingangs bereits erwähnt, ein barrierefreier Zugang zu ihren Angeboten.

Wie schon im ersten Schwerpunktheft $\mathrm{zu}$ dieser Reihe finden Sie auch in diesem Band einen wissenschaftlichen Artikel zu Intersexualität und Differences of Sexual Development (DSD). Im Artikel von P.-M. Holterhus werden eingehend die biologischen Grundlagen zur Intersexu- 
alität sowie diagnostische Möglichkeiten dargestellt. Der Autor verdeutlicht einmal mehr eine Tatsache, die in nahezu allen Themenbereichen der sexuellen und reproduktiven Gesundheit eine übergeordnete Rolle spielt: Sowohl die Diagnose als auch die verschiedenen Betreuungs- und Therapieansätze setzen erfahrene, multidisziplinäre Teams voraus. Ohne Interdisziplinarität sind die komplexen Fragen der modernen Medizin nicht zu lösen.

Liebe Leserinnen und Leser, wir wünschen Ihnen eine spannende und erkenntnisreiche Lektüre.

Ihre

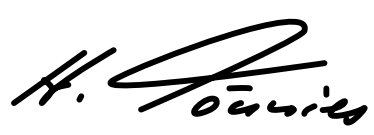

H. Tönnies

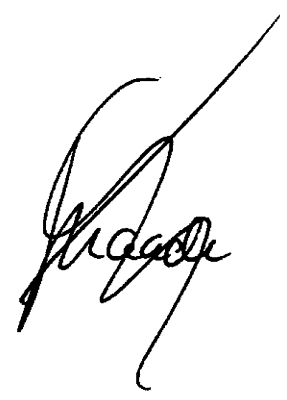

L. Schaade

\section{Korrespondenzadresse}

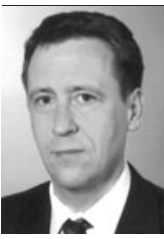

PD Dr. H. Tönnies

Geschäftsstelle GendiagnostikKommission,

Robert Koch-Institut

DGZ-Ring 1, 13086 Berlin

toenniesh@rki.de

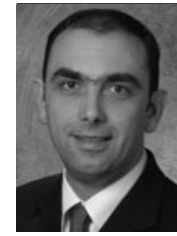

PD Dr. L. Schaade

Zentrum für Biologische

Gefahren und Spezielle Patho-

gene; Vizepräsident,

Robert Koch-Institut

Nordufer 20, 13353 Berlin

schaadel@rki.de

Interessenkonflikt. H. Tönnies und L. Schaade geben an, dass kein Interessenkonflikt besteht. 